\title{
Les noms généraux sentiment et émotion
}

\section{The French Nouns sentiment and émotion}

\author{
Iva NOVAKOVA, Julie SORBA, Agnès TUTIN \\ Univ. Grenoble Alpes, LIDILEM, F-38000 Grenoble, France
}

\section{Résumé}

Cet article propose une analyse des deux noms sentiment et émotion dans deux corpus : journalistique et littéraire. Contrairement à leur apparente similitude, sentiment et émotion fonctionnent très différemment au plan syntaxique, sémantique et discursif. Nous postulons que la comparaison des deux genres discursifs doit nous permettre de mieux appréhender leur statut de noms généraux. Nous proposons d'éclairer l'analyse synchronique par une étude diachronique ponctuelle (corpus romanesque de Frantext). Notre objectif est ainsi de comprendre, au regard de l'évolution diachronique des deux termes, pourquoi et comment sentiment est devenu un classifieur et un shell noun, comparé à émotion.

Mots clés français : noms généraux, sentiment, émotion, classifieur, shell noun

\section{Abstract}

In this paper, we focus on the two French nouns sentiment and émotion in order to analyze their linguistic properties in two corpora (journalistic and literary). Even if they look alike, sentiment and émotion work very differently at the syntactical, lexical and discursive levels. We also propose to shed light on the synchronic analysis by a diachronic study based on the Frantext corpus of French novels. Our objective is thus to understand, in the light of the diachronic evolution, why and how sentiment has become a classifier and a shell noun, what is not the case for émotion.

Key words: general nouns, feeling, emotion, classifier, shell noun

\section{INTRODUCTION}

Les noms sentiment et émotion font partie, selon Plantin (2011), des sept termes génériques dans le vocabulaire des émotions : pathos, humeur, passion, sentiment, éprouverléprouvé, affect, émotion. Toutefois, sentiment, dont l'origine est interne, c.à.d. prenant sa source dans l'individu lui-même, a un sens plus large et moins exclusivement associé aux affects (Tableau 1), comparé à émotion, dont le stimulus est externe et qui se présente de ce fait comme un affect provoqué.

Tableau 1 : Les sens de sentiment d'après les outils lexicographiques (PR, TLF)

\begin{tabular}{|l|l|l|}
\hline domaine de l'affectivité & un sentiment de joie & synonyme : émotion \\
\hline domaine des sens & un sentiment d'engourdissement & sens étymologique (sentir) \\
\hline domaine de l'intellect & un sentiment de justice & avoir l'impression \\
\hline
\end{tabular}

Les acceptions attachées à ces trois domaines (affectivité, sensation, intellect) sont répertoriées dans les premières attestations de sentiment. Dérivé du verbe esmouvoir, le nom émotion, pour sa part, désigne originellement un «mouvement assez vif ». Son sens affectif se stabilise aux XVII ${ }^{e}$ et XVIII' ${ }^{\mathrm{e}}$ s. et son emploi devient fréquent. Selon Ferrer et Ramond (2017: 8), «sa présence est liée à l'émergence de l'individu moderne et conjointement à l'intérêt suscité par les intermittences de la conscience et l'exploration de la vie psychique ».

Dans les typologies linguistiques des noms d'affect (p. ex. Anscombre, 1995, Buvet et al., 2005, Tutin et al., 2006), sentiment et émotion sont utilisés comme des termes permettant d'identifier des classes de noms. En revanche, peu de travaux ont été véritablement consacrés, à notre connaissance, à l'emploi de ces deux lexies comme noms classifieurs (p. ex. Krzyżanowska, 2014 ; Augustyn \& Grossmann, 2014, travaux privilégiant la perspective contrastive ou sémantico-syntaxique). Dans cette étude, menée dans la continuité du projet Emolex ${ }^{1}$, nous aimerions aller plus loin. Afin de clarifier le statut des deux noms, nous retenons une série de paramètres de différente nature :

a) Nous analysons les deux noms en synchronie, en comparant leurs propriétés lexico-syntaxiques et discursives.

b) Nous comparons le fonctionnement de sentiment et émotion dans deux genres discursifs (corpus journalistiques et littéraires) afin de mieux appréhender leur statut.

\footnotetext{
${ }^{1}$ Le projet franco-allemand Emolex (2010-2013, http://emolex.u-grenoble3.fr/) a eu comme objectif de classer et d'analyser le lexique des émotions dans cinq langues européennes.
} 
c) Nous relions les propriétés linguistiques établies en synchronie à l'évolution diachronique des deux lexies, ce qui, à notre connaissance, n'a pas été fait de manière systématique dans les études existantes.

Nous faisons l'hypothèse qu'émotion est un «nom général » au sens de Halliday et Hasan (1976), c'est-à-dire comme un hyperonyme large avec un sens affectif stable et bien circonscrit, à la différence de sentiment qui fonctionne davantage comme un «shell noun» ('nom coquille') (Schmid 2000) et qui prend sa valeur sémantique en contexte, du fait d'une plus grande plasticité sémantique. Nous visons ainsi à comprendre, au regard de l'évolution diachronique des deux termes, pourquoi et comment sentiment est devenu un terme classifieur et un shell noun, ce qui n'est pas le cas d'émotion.

\section{METHODOLOGIE, CORPUS}

Le corpus utilisé pour l'étude synchronique est issu de 1 'EmoBase ${ }^{2}$ : il est constitué de deux échantillons équilibrés de textes journalistiques (Libération 2007-2008) et de textes littéraires romanesques contemporains. Les données diachroniques sont issues de la base de données Frantext (version janvier 2017). Le corpus de travail a été sélectionné dans le genre littéraire « roman» qui comprend 549 textes (soit $51.5 \mathrm{M}$. de mots) répartis du XVI au XIXe siècle ${ }^{3}$. Les corpus sont présentés dans le Tableau 2 : à côté de la fréquence absolue des deux lexies (nombre d'occurrences dans chaque sous-corpus), nous indiquons la fréquence relative (nombre d'occurrences pour un million de mots dans chaque sous-corpus).

Tableau 2 : Données de l'étude dans les corpus synchronique et diachronique

\begin{tabular}{|c|c|c|c|}
\hline & Millions de mots & sentiment & émotion \\
\hline Frantext XVI ${ }^{\mathrm{e}} \mathrm{s}$. & 1.4 & 38 occ. (27) & 6 occ. (4) \\
\hline Frantext XVII ${ }^{\mathrm{e}} \mathrm{s}$. & 3.8 & 300 occ. $(79)$ & 69 occ. (18) \\
\hline Frantext XVIII' $\mathrm{s}$. & 13.5 & 4286 occ. $(317)$ & 854 occ. (63) \\
\hline Frantext XIX $\mathrm{s}$ & 32.8 & 7207 occ. (219) & 4276 occ. (130) \\
\hline EmoBase corpus littéraire $\mathrm{XX}-\mathrm{XX} \mathrm{I}^{\mathrm{e}} \mathrm{s}$. & 16 & 870 occ. (54) & 1562 occ. (97) \\
\hline EmoBase corpus journalistique $\mathrm{XXI}^{\mathrm{e}} \mathrm{s}$. & 16 & 586 occ. (36) & 900 occ. $(56)$ \\
\hline
\end{tabular}

Notre méthodologie allie l'étude systématique de la combinatoire lexico-syntaxique des noms (Blumenthal, 2009, Novakova \& Tutin, 2009) à des méthodes lexico-statistiques (Blumenthal, 2012, Kraif \& Diwersy, 2014). L'analyse fonctionnelle de sentiment et émotion proposée ici s'inspire du contextualisme britannique (Sinclair, 2004, Hoey 2005). Enfin, dans la continuité de Condamines (2005), nous étudions le fonctionnement anaphorique des deux lexies, en particulier dans l'anaphore nominale démonstrative. Afin de mieux appréhender leur statut dans les deux corpus, nous analysons d'abord leur profil syntaxique (section 3), puis leur profil lexical (section 4) et, enfin, leur profil discursif, en particulier, les phénomènes d'anaphore (section 5).

\section{LE PROFIL SYNTAXIQUE DE SENTIMENTET D'EMOTION}

Les travaux de Schmid (2000 : 22-23), à la suite de Winter (1992), ont montré que le sens des shell nouns comme idea ou evidence était surtout actualisé en contexte, à l'aide de compléments phrastiques (idea that/to/wh) ou dans des configurations comme idea that/to/wh qui en déterminent l'extension (Cf. Legallois, 2006 pour une adaptation au français). Qu'en est-il pour émotion et sentiment ?

L'étude des profils syntaxiques fait apparaître en synchronie des structures syntaxiques fort différentes pour les deux noms. En effet, dans les corpus synchroniques, émotion apparaît souvent sans modifieur (dans les titres, phrases averbales, constructions attributives, structures à présentatif) avec un léger avantage, pour ces distributions, dans le corpus journalistique $(8,2 \%)$ comparé au littéraire $(6,5 \%)$ :

(1) Il y a tant d'émotion dans son art. [...] (Libération, 25/08/2007).

(2) Aucune empathie, aucune émotion pour l'autre. (Chattam, Prédateurs, 2007)

On peut parler ici d'émotion «à l'état pur » (Novakova \& Sorba, 2014 : 164).

Sentiment, quant à lui, est moins présent dans ces structures (4,6\% dans le journalistique et $4 \%$ dans le littéraire). Lorsqu'on le retrouve dans des phrases averbales ou des structures à présentatif (il y $a$ ), il introduit un autre nom psychologique ${ }^{4}$ (3) ou a le sens d'impression' (4) :

(3) Un sentiment de solitude, d'isolement (Libération, 25/07/2007)

${ }^{2}$ La base de données EmoBase constituée dans le cadre d'Emolex est librement consultable à l'adresse suivante : http://emolex.u-grenoble3.fr/emoBase/.

${ }^{3}$ Pour les besoins de l'enquête et de l'analyse diachroniques, nous avons retenu un découpage traditionnel par siècle, malgré les inconvénients épistémologiques d'une telle organisation (Soutet, $2011: 11$ ).

${ }^{4}$ Cet emploi est bien attesté au XIX ${ }^{\mathrm{e}} \mathrm{s}$ : : C'est ce sentiment exquis de pudeur et de dévouement qui dominait chez eux (Sand, Valentine, 1869). 
(4) Parfois, aussi, il y a chez lui le sentiment d'être né trop tard, dans un monde trop vieux. (Libération, 03/02/2017)

Dans le corpus diachronique, les phrases averbales ou les structures à présentatif, totalement absentes aux XVI ${ }^{e}$ et XVII ${ }^{e}$ siècles, apparaissent pour les deux noms seulement de manière sporadique (moins de $1 \%$ ) aux XVIII et XIX ${ }^{\mathrm{e}} \mathrm{s}$. Le développement progressif de ces structures s'explique probablement par les évolutions esthétiques qui affectent le roman à partir du XVIII ${ }^{\mathrm{e}} \mathrm{s}$. avec la mise en scène du sujet ému et l'irruption de la parole émotive et bouleversée (Ramond 2017).

En synchronie, sentiment apparaît très souvent ( $40 \%$ des cas, contre $1 \%$ pour émotion) dans les deux corpus avec une complémentation de type nominal (SN prep), phrastique (que P) ou verbal (de Vinf) ${ }^{5}$. Lorsqu'il est suivi d'un SN prép, ce nom est classifieur $(5,6)$ : il sert à introduire d'autres noms psychologiques (sentiment d'inquiétude, d'abandon), distribution qu'il ne partage pas avec émotion (*émotion d'inquiétude, d'abandon) :

(5) Donc j'éprouve vis-à-vis de votre personne un sentiment de méfiance. (Dard, Du sable dans la vaseline, 1998)

(6) Dans mon boulot, on a un sentiment de ras-le-bol. (Libération, 19/01/2007)

Dans le corpus diachronique, la situation est légèrement différente. En effet, dans les romans des XVI et XVII ${ }^{\mathrm{e}}$ s., émotion et sentiment sont abondamment pourvus d'une complémentation de type nominal (sentiment d'amour) et, contrairement à ce qui est observé en synchronie, émotion peut même être suivi d'un SN prep introduisant un autre nom d'affect ou psychologique (émotion d'une colère). Cela peut s'expliquer par le sens premier d'émotion («mouvement vif »). Néanmoins, dès le XVI ${ }^{\mathrm{e}} \mathrm{s}$., sentiment utilise essentiellement des noms de qualité comme complément (sentiment de vertu, de piété). Dans les romans du XVIII et du XIX ${ }^{\mathrm{e}}$ s., la complémentation en $d e \_\mathrm{N}$ devient une tournure nettement privilégiée par sentiment (14\% et $24 \%$ ) plutôt que par émotion (6\% et 9\%) :

(7) son âme fut pénétrée d'un sentiment d'amour et de reconnaissance pour le ciel. (de Staël, Corinne ou l'Italie, 1807)

Par ailleurs, lorsqu'il régit une complémentation du type que $\mathrm{P}$ (complétive) ou de Vinf, sentiment se trouve essentiellement dans des constructions à verbe support avoir, donner le sentiment que $\mathrm{P}$ ou de Vinf $(8,9)$. Dans ce cas, il signifie 'avoir l'impression de' et son sens s'éloigne du sens affectif :

(8) Alors que les Français d'origine africaine ont le sentiment qu'une injustice a été commise à l'égard de leurs aïeux. (Libération, 10/02/2007)

(9) Son visage s'éclaira. Elle parut se tranquilliser. Elle me libéra. [...] J'eus le sentiment d'avoir posé la bonne question. (Boulle, La Planète des singes, 1963)

Dans le corpus diachronique, la complémentation du type que $\mathrm{P}$ ou de Vinf n'est attestée que pour sentiment et de manière très sporadique (moins de $1 \%$ des occurrences dans les romans du XIX ${ }^{\mathrm{e}} \mathrm{s}$.).

L'étude des distributions syntaxiques des deux noms corrobore le constat de Blumenthal (2009: 55) selon lequel un nom sémantiquement peu saturé (ici, sentiment) nécessite une complémentation plus fournie, alors qu'un nom hautement saturé (ici, émotion) a une complémentation plus réduite ${ }^{6}$. De plus, la complémentation nominale, phrastique ou verbale «qui bloque l'interprétation de sentiment comme 'état affectif' » (Augustyn \& Grossmann, $2014: 102$ ) montre qu'il fonctionne comme un shell noun et permet de circonscrire son extension sémantique, tout particulièrement dans son acception d' 'impression' ou 'sensation'. L'étude diachronique confirme que ce statut a été progressivement acquis par sentiment. En revanche, émotion se comporte davantage comme un nom sémantiquement plus plein, de sens affectif, et n'occupe plus la fonction de nom classifieur, désormais dévolue à sentiment.

\section{LE PROFIL LEXICAL DE SENTIMENT ET D'EMOTION}

Notre méthodologie permet aussi d'identifier les associations lexicales statistiquement spécifiques de sentiment et émotion dans les deux genres (journalistique et littéraire) pour établir leur profil lexical. La spécificité des associations de ces lexies avec leurs collocatifs ( «accompagnateurs préférentiels », Blumenthal 2012) est calculée selon l'indice loglikelihood ratio (LLR, Dunning 1993) ${ }^{7}$. Le tableau 3 présente les dimensions sémantiques ${ }^{8}$ retenues pour l'analyse des associations lexicales :

Tableau 3. Dimensions sémantiques

\begin{tabular}{|l|l|}
\hline Dimensions sémantiques & Collocations \\
\hline expérienciation & ressentir une émotion ; éprouver un sentiment \\
\hline Manifestation & trembler d'émotion \\
\hline Contrôle & dissimuler son émotion \\
\hline
\end{tabular}

\footnotetext{
${ }^{5}$ Seule se rencontre avec émotion la complémentation (de Vinf) et ce, dans moins de $1 \%$ des cas.

${ }^{6}$ C'est aussi une caractéristique relevée pour d'autres shell nouns se caractérisant par la pauvreté de leur contenu descriptif comme fait, idée, hypothèse (Schmid, 2000 ; Legallois, 2006 ; Huyghe, 2015).

${ }^{7}$ Il s'agit du calcul du rapport de vraisemblance qui permet d'évaluer si une association lexicale s'écarte significativement d'une distribution aléatoire ou non. Le seuil de spécificité des associations lexicales (LLR) a été fixé à 10,83.

${ }^{8}$ Ces dimensions sémantiques ont été établies à partir des traits sémantiques des collocatifs spécifiques des noms d'affects. Pour plus de détails à ce sujet, Diwersy et al. (2014).
} 
Nous proposons d'analyser en détail les « attirances » ou les « aversions » spécifiques (Hoey 2005) des deux noms pour les collocatifs renvoyant à chacune de ces dimensions dans les corpus journalistique et littéraire.

\subsection{L'expérienciation}

Dans le corpus littéraire, un/le sentiment de a une préférence très marquée pour le verbe éprouver (LLR 821) alors que, dans le corpus de presse, cette structure attire préférentiellement les verbes supports avoir (LLR 870) et donner (LLR 481). Or, comme le montrent les exemples, les collocations de ce type (avoir/donner le sentiment de) n'ont pas de sens affectif et signifient 'avoir/donner l'impression de'. Dans ces combinaisons, sentiment fonctionne comme un terme générique dont l'acception relève du domaine de l'intuition' :

(10) Avez-vous le sentiment de maîtriser votre sujet ? (Libération, 24/01/ 2007)

Dans les deux corpus, un/le sentiment de est suivi de noms d'affect (regret, angoisse, sympathie) mais surtout de noms psychologiques

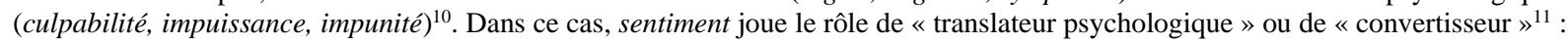
on $\mathrm{n}$ '*éprouve pas une impunité mais bien un sentiment de $\sim$. Autrement dit, les noms psychologiques basculent dans le domaine affectif grâce au classifieur sentiment. Ce rôle est attesté pour sentiment dès les romans du XVII ${ }^{\mathrm{e}} \mathrm{s}$. (un sentiment de vertu, de vengeance, d'injustice). Ainsi, nos résultats confirment que «sentiment de ne peut être considéré comme classifiant prioritairement les sentiments en tant qu'affects : il s'applique à toute la palette du ressenti psychologique, depuis la sensation jusqu'à la saisie intellectuelle, en passant par le ressenti affectif » (Augustyn \& Grossmann, 2014 : 132). Il s'agit d'un terme « couvrant » qui conserve les trois sens attestés en diachronie (intellect, sensations, affectivité, cf. Tableau 1.).

Quant à émotion, il attire préférentiellement le verbe support ressentir ${ }^{12}$ (LLR 250) dans le corpus littéraire moderne comparé au corpus journalistique :

(11) Mais, depuis peu, Dempsey ressentait une émotion nouvelle au fond de son cœur [...]. (Robillard, Les Dragons de l'Empereur Noir, 2003)

Sans discontinuité depuis les romans du XVI e s., émotion s'emploie avec sentir (12) et sentiment avec avoir (13) :

(12) dens mon estomach je sen ne sçay quelle divine esmotion qui me contrainct à prophetiser. (Flore, Contes amoureux, 1537)

(13) Aussy, le cueur de l'homme, qui n'a nul sentiment d'amour aux choses visibles. (Navarre, L'Heptaméron, 1550)

Au fil du temps, les constructions à verbe support se sont étendues pour les deux lexies. Par exemple, on trouve couramment avoir en compagnie d'émotion dès le XVII ${ }^{\mathrm{e}} \mathrm{s}$. (14), alors que cette association a disparu dans le corpus contemporain. D'autre part, éprouver apparaît dans les romans du XVIII ${ }^{\mathrm{e}} \mathrm{s}$. avec les deux noms $(15,16)$ avant de se spécialiser comme accompagnateur privilégié de sentiment en synchronie :

(14) je n'ay nulle émotion que celle d'un plaisir innocent. (Abbé de Pure, La Prétieuse ou le Mystère des ruelles, 1656)

(15) j'éprouvai cette vive et délicieuse émotion que me donnoit quelquefois sa présence inattendue. (Rousseau, La Nouvelle Hélö̈se, 1761)

(16) nous sommes toujours redevables à ceux qui nous font éprouver un sentiment doux. (Graffigny, Lettres d'une jeune Péruvienne, 1747)

L'étude diachronique révèle ainsi l'ancienneté de l'association sentir + émotion tandis que la combinaison de éprouver + sentiment semble plus récente.

En bref, les verbes supports éprouver, ressentir, avoir et donner permettent de distinguer sentiment et émotion et ce, dans les deux types de corpus, pour la dimension expérienciation. La combinatoire différente reflète donc un statut différent pour les deux noms.

\subsection{La manifestation et le contrôle}

Le nom émotion attire préférentiellement des collocatifs pour la manifestation ou le contrôle (Tableau 4) que l'on ne retrouve pratiquement pas, dans les corpus modernes, pour sentiment :

Tableau 4 : Associations lexicales spécifiques pour émotion (dimensions manifestation et contrôle)

\begin{tabular}{|l|l|c|}
\hline \multicolumn{1}{|c|}{ Dimension } & \multicolumn{1}{|c|}{ Associations lexicales spécifiques } & LLR \\
\hline \multirow{3}{*}{ manifestation } & trembler d'émotion & 119 \\
\cline { 2 - 3 } & manifester son émotion & 64 \\
\hline \multirow{4}{*}{ Contrôle } & se remettre de son émotion & 158 \\
\cline { 2 - 3 } & émotion contenue & 99 \\
\cline { 2 - 4 } & trahir son émotion & 96 \\
\cline { 2 - 4 } & masquer son émotion & 89 \\
\hline
\end{tabular}

\footnotetext{
${ }^{9}$ Cf. aussi Augustyn \& Grossmann (2014) ; Krzyżanowska (2014).

${ }^{10}$ Ces noms sont rangés par ordre décroissant de l'indice LLR.

${ }^{11}$ Nous empruntons ces termes à Augustyn \& Grossmann (2014 : 127).

${ }^{12} \mathrm{Cf}$. aussi à ce sujet Blumenthal (2009:49).
} 
Ces associations sont surreprésentées dans le corpus romanesque, ce qui pourrait s'expliquer par le fait que dans ce genre littéraire la description des personnages et de leurs comportements occupe une place de choix (personnages qui tentent de maîtriser ou au contraire d'exprimer ou de manifester leurs émotions) :

(17) Tremblant d'émotion contenue, j'approchai ma main de son épaule et effleurai son épiderme fin. (Werber, Les Thanatonautes, 1994).

L'exemple (17) illustre les deux dimensions sémantiques (manifestation et contrôle) générées par les collocatifs d'émotion.

En revanche, le seul collocatif spécifique de sentiment pour la dimension contrôle et, en particulier, pour sa valeur 'absence de contrôle' (Diwersy et al. 2014) est envahir (LLR 64) (être envahi par un sentiment de tristesse, de culpabilité, d'impuissance) et ce, uniquement dans le corpus littéraire contemporain (18). Cette association spécifique semble s'amorcer au XIX ${ }^{\mathrm{e}} \mathrm{s}$. (19) car le corpus romanesque ancien n'en porte pas trace auparavant :

(18) $[\ldots]$ je me sentis soudain envahi par un bizarre sentiment de nostalgie. (Boulle, La Planète des singes, 1963)

(19) L'horrible sentiment des abîmes l'avait envahie. (Balzac, Séraphita, 1846).

L'analyse du corpus diachronique révèle une partition plutôt nette concernant ces deux dimensions sémantiques entre les occurrences des $\mathrm{XVI}^{\mathrm{e}}$ et $\mathrm{XVII}{ }^{\mathrm{e}}$ s., d'une part, et celles des siècles suivants, d'autre part. En effet, sentiment et émotion entrent dans un premier temps quasi-exclusivement en combinaison avec des termes indiquant le contrôle (dissimuler, vaincre, combattre, cacher, surmonter + émotion et rejeter, cacher, retenir + sentiment). Puis, à partir du XVIII ${ }^{\mathrm{e}} \mathrm{s}$. et plus largement au $\mathrm{XIX}^{\mathrm{e}} \mathrm{s}$., sans perdre ce profil combinatoire ${ }^{13}$, ils développent d'autres associations avec des termes relevant de la dimension manifestation (laisser paraitre, s'empourprer, trace, air, marque de + émotion et feindre, étaler, témoigner + sentiment). Ces faits linguistiques témoignent sans doute de phénomènes culturels et littéraires : la retenue émotionnelle installant progressivement une civilisation des mœurs à l'époque moderne ${ }^{14}$, les émotions doivent être contrôlées ; puis les affects s'affichent de nouveau dans le mouvement romantique qui submerge l'Europe au XIX ${ }^{\mathrm{e}}$ siècle ${ }^{15}$.

Les deux dimensions (contrôle et manifestation) sont donc spécifiques à émotion, affect que l'on manifeste ou qu'on essaie de maitriser. Elles sont beaucoup moins caractéristiques pour sentiment, ce qui pourrait s'expliquer par sa tendance dominante à évincer le sens affectif et à fonctionner comme un shell noun.

En résumé, la combinatoire lexicale des deux noms, observée dans les deux corpus, permet de mieux cerner leur profil lexical et de les distinguer. Sentiment et émotion ont chacun des collocatifs spécifiques pour la dimension expérienciation : sentiment attire préférentiellement les verbes supports (avoir, donner) et prend ainsi le sens de 'avoir/donner l'impression de' ; émotion, quant à lui, attire de nombreux collocatifs pour les dimensions manifestation et contrôle dans le corpus littéraire. Ce dernier fonctionne ainsi essentiellement comme nom d'affect. Les différences relevées dans les corpus journalistique et littéraire s'avèrent éclairantes pour appréhender le fonctionnement de ces deux noms.

\section{LE PROFIL DISCURSIF DE SENTIMENTET D’EMOTION : LES EMPLOIS ANAPHORIQUES}

Sur le plan discursif, les noms généraux, du fait de leur contenu sémantique large, sont souvent employés dans des expressions anaphoriques renvoyant à un référent nominal (ex : joie ... émotion); les shell nouns, quant à eux, renvoient à des propriétés ou à des faits apparaissant dans des entités textuelles plus larges. Par leur contenu dénotatif assez vague (ex : problème, fait), ils ont précisément pour caractéristique de prendre leur valeur fonctionnelle en contexte, en lien avec le contenu textuel antérieur ou ultérieur.

Dans notre travail de comparaison entre émotion et sentiment, nous faisons l'hypothèse que le nom sentiment sera plus productif comme anaphore, en particulier dans les contextes d'anaphores résomptives. Dans cette perspective, nous avons extrait les associations $c e(s)+$ émotion $(s) /$ sentiment $(s)$, une configuration qui apparaît, de manière générale, plus caractéristique des emplois anaphoriques que le syntagme défini.

\subsection{La fréquence des structures $c e+\mathrm{N}$}

Conformément à nos attentes, la productivité de la structure $c e+\mathrm{N}$ est bien plus grande avec sentiment (10\% des occurrences sur les 1456 occ.), en particulier au singulier, qu'avec émotion (2,5\% des occurrences sur 2462). En outre, on observe une différence nette liée au genre, la structure étant plus récurrente dans la littérature que dans la presse (émotion : $77 \%$ dans la littérature et 23\% dans la presse ; sentiment : $62 \%$ pour la littérature et $38 \%$ dans la presse).

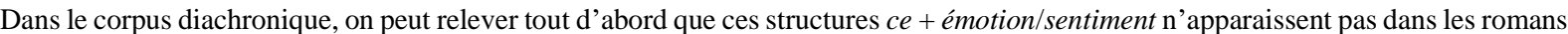
du XVI ${ }^{\mathrm{e}}$ s. Au XVII ${ }^{\mathrm{e}}$ s., ce sont les séquences au singulier qui sont les plus fréquentes (18\% singulier vs $3.5 \%$ pluriel); de plus, $c e(s)$ émotion $(s)$ est largement plus employé $(15 \%)$ que $c e(s)$ sentiment $(s)(6.5 \%)$. Étrangement, au XVIII ${ }^{\mathrm{e}} \mathrm{s}$., les romanciers délaissent ce tour anaphorique pour les deux noms (moins de 1\%). Enfin, c'est au XIXe s. que s'amorce la tendance observée dans le corpus

\footnotetext{
${ }^{13}$ Sur la notion de profil combinatoire, cf. Blumenthal (2009).

${ }^{14} \mathrm{C}$ 'est la théorie traditionnelle défendue par Elias qui distingue ainsi le Moyen-âge de l'époque moderne (voir Rosenwein et al. $2006: 33)$.

${ }^{15}$ Ariès \& Duby parlent d'une « véritable réinvention du sentiment » au XIX ${ }^{\mathrm{e}}$ s. (1987 : 522).
} 
synchronique à savoir l'emploi plus fréquent de $c e(s)$ sentiment $(s)(8 \%$ vs $6 \%$ pour $c e(s)$ émotion $(s))$ avec une productivité plus élevée pour la séquence au singulier (6\%) qu'au pluriel (2\%).

\subsection{Les valeurs du SN démonstratif}

La structure $c e+\mathrm{N}$ a plusieurs valeurs, comme déjà observé ailleurs (cf. par exemple Cornish 2008). Nous ne retenons ici que celles qui sont pertinentes pour l'étude discursive de nos deux noms. Comme nous le verrons, ces emplois sont anciens.

\subsubsection{Emploi anaphorique coréférentiel nominal}

Dans ce premier cas, le SN démonstratif renvoie à un référent nominal introduit préalablement, qu'il s'agisse d'une anaphore fidèle (avec même contenu lexical du type émotion $\rightarrow$ cette émotion) ou d'une anaphore infidèle (peur $\rightarrow$ cette émotion). Dans l'exemple (20), l'emploi anaphorique ces sentiments renvoie ainsi à aucune peur, aucune colère :

(20) L'animal se cambra et hurla [...]. Diane n'éprouvait aucune peur, aucune panique. Elle se situait au-delà de ces sentiments. (Grangé, Le Concile de pierre, 2000)

Dans le corpus synchronique, les emplois coréférentiels de $c e+$ sentiment sont trois fois plus fréquents que ceux de $c e+e ́ m o t i o n:$ il apparaît à la fois dans les acceptions d' 'affect', souvent dans la littérature, ou d' 'impression/point de vue', davantage dans la presse. Si quelques anaphores fidèles apparaissent (majoritairement dans la presse, $11 \mathrm{occ}$. ), on relève surtout de nombreuses anaphores infidèles avec hyperonymie (34 occ.). Ce + émotion apparaît également possible en anaphore fidèle (6 occ.), comme dans l'exemple (21) ou infidèle (principalement, en relation d'hyperonymie, 4 occ.) :

(21) Il ne s'agit pas pour autant de suggérer que l'émotion de Nicolas Sarkozy serait feinte [...]. Je crois que cette émotion fonctionne d'autant mieux politiquement qu'elle est sans doute sincère. (Libération, 25/10/2007)

Dans les romans du XVII $\mathrm{e}$ s., on rencontre ce + émotion en anaphore fidèle ou infidèle (22) :

(22) il fut tellement esmeu de l'excez de joye, que la presence de Maistresse luy causa, qu'il en eut la fiévre bien violente, et cet accident retarda de beaucoup sa guerison : mais laissons-le reparer par un grand repos, le mal que cette émotion luy donna, jusques à ce qu'il guerisse entierement. (Boisrobert, Histoire indienne d'Alexandre et d'Orazie, où sont entremeslées les aventures d'Alcidaris, de Combaye et les amours de Pyroxène, 1629).

Dans le corpus diachronique, cet emploi n'est pas inconnu mais il demeure rare.

\subsubsection{Emploi de deixis textuelle}

Cette valeur d'anaphore résomptive apparaît quand le SN anaphorique résume un pan textuel, fonctionnant comme un pointeur métadiscursif, ou renvoie à une notion introduite par une proposition. Dans l'exemple (23), ce sentiment renvoie à tout le contenu textuel correspondant à l'affect :

(23) Interrogé il y a vingt ans [...] Kapuscinski répondait : «L'autre jour, j’ai regardé les résultats des prix littéraires en France. C'était incroyable. Pas un seul livre n'avait à voir avec la réalité du monde qui nous entoure. » Luc de Goustine, qui m'a accueilli chez lui en Corrèze lors de mon dernier voyage à vélo, partage ce sentiment. (Libération, 29/12/2007)

Dans le corpus synchronique, cet emploi est très fréquent pour sentiment, que le nom renvoie à un affect (42 occ.), à une impression ou à un point de vue (26 occ.). Il est aussi attesté dès les romans du XVII ${ }^{\mathrm{e}}$ s. (24) :

(24) en ce moment-là il s'imagina que puisque dom Manuël sçavoit le dessein du roy, il l'avoit fait sçavoir à Mathilde qui peutestre y consentoit, et dans ce sentiment il estoit prest d'expirer de douleur. (Scudéry, Mathilde, 1667)

En revanche, cet emploi est plus rare pour émotion dans le corpus synchronique, qui apparaît dans ce cas plutôt au pluriel (25) :

(25) Et un peu de verre, qui provenait du carreau brisé, parmi les documents posés sur le bureau. Apparemment, celui qui est venu vous agresser cherchait quelque chose, ou tout du moins à vérifier si vous saviez quelque chose. Annabel se redressa. Au milieu de toute cette agitation et de ces émotions, elle ne s'était pas encore posé la question essentielle. (Chattam, Maléfices, 2004)

Dans l'exemple (25), le nom émotion ne renvoie pas strictement à un affect, mais plutôt aux événements où des « émotions fortes » ont été vécues. La portée de l'anaphore dépasse ici les quelques phrases précédentes et renvoie à des éléments antérieurs, qui peuvent être difficiles à identifier dans le texte. La présence d'expressions préfabriquées (toutes ces émotions, se remettre de ces émotions, etc.) peut donner l'impression d'un cliché narratif. L'emploi est ancien mais n'est cependant pas très répandu dans le corpus diachronique (26) :

(26) Il jeta au hasard dans une malle ses habits, son linge, tous ses papiers ; il ramassa tous les souvenirs de Marie, éparpillés par l'ouragan de la colère paternelle, les enveloppa soigneusement, et ayant fait monter le cocher, il lui fit transporter sa malle dans la voiture. En descendant l'escalier bien lentement, car il était faible et brisé par toutes ces émotions, il rencontra son père. (Murger, Scènes de la vie de jeunesse, 1851)

En bref, l'emploi de deixis textuelle apparaît principalement avec sentiment, conformément à son statut de shell noun mais peu fréquemment avec émotion, sauf dans l'acception événementielle spécifique. 


\subsubsection{Emploi « emphatique »}

L'emploi emphatique, non anaphorique, met en jeu des dimensions interpersonnelles et des connaissances partagées de type culturel ou encyclopédique, sans renvoyer à un référent préalablement introduit. Cet emploi est souvent lié à la structure de classifieur $c e$ sentiment de $\mathrm{N}$, déjà décrit à la section 4.1, et semble fréquemment utilisé dans les descriptions.

(27) Tu vois ce que je veux dire ? Elle avançait maladroitement, cherchant ses mots pour expliquer ce sentiment entre nostalgie et peur qu'elle-même ne comprenait pas très bien. (Chattam, L'Âme du mal, 2002)

(28) Les pères ne veulent-ils donc que du respect ? Leur donne-t-il même tout ce qu'on leur doit ? Et ne leur devrait-il pas être bien doux de voir la reconnaissance augmenter et affermir, dans le cœur de leurs enfants, ce sentiment d'amour que la nature y a déjà gravé ? (Crébillon, Les Égarements du cœur et de l'esprit, 1738)

Ce dernier type est fréquent dans le corpus synchronique avec le nom sentiment (à peu près un quart des emplois, équitablement répartis entre les deux genres). Dans ce cas, le SN démonstratif comporte généralement une expansion (relative, adjectivale ou complément de nom à fonction appositive). À l'inverse, cet emploi est rare avec émotion.

En bref, l'analyse synchronique du comportement discursif de ce + sentiment et ce + émotion confirme la différence de statut de ces deux noms génériques dont nous indiquons la répartition dans le Tableau 5.

Tableau 5 : Répartition des emplois de ce $N$ dans le corpus synchronique

\begin{tabular}{|l|l|l|}
\hline & Cette émotion/ces émotions & Ce sentiment/des émotions \\
\hline Anaphore coréférentielle nominale & $24,4 \%$ & $22,4 \%$ \\
\hline Deixis textuelle & $7,3 \%$ & $33,8 \%$ \\
\hline Emplois emphatiques & $7,3 \%$ & $25,8 \%$ \\
\hline Autres & $61 \%$ & $18 \%$ \\
\hline
\end{tabular}

Sentiment est plus fréquent comme anaphore nominale coréférentielle, et apparaît préférentiellement dans deux configurations : a) comme nom anaphorique « résomptif » renvoyant à des contenus textuels larges, ce qui souligne son statut de shell noun; b) dans la configuration «emphatique». Dans le corpus diachronique, nous avons pu constater que ces deux configurations sont attestées de manière ancienne. Émotion dans son sens d'affect a un emploi discursif plus limité : peu fréquent, il apparaît essentiellement en anaphore fidèle et infidèle, et n'est pas susceptible d'emplois « emphatiques ». On relève, en revanche, que son acception d'événement est susceptible d'un emploi de deixis textuelle, le rapprochant ainsi, mais uniquement dans cette acception spécifique, d'un shell noun.

\section{CONCLUSION ET PERSPECTIVES}

Pour récapituler, sentiment et émotion sont deux noms généraux du domaine du vocabulaire affectif mais de statut différent.

Le faible degré de saturation sémantique de sentiment entraîne des structures actancielles et des complémentations plus fournies. Il a également un important rôle de liage et de cohésion sur le plan discursif. Ces propriétés le rapprochent des 'noms coquilles' (Schmid 2000), en particulier dans son acception d' 'impression', où il acquiert son contenu sémantique en contexte, de la même façon que le nom feeling, intégré par Schmid (2000) dans la classe des noms psychologiques (sous-classe des noms mentaux). Dans son acception de nom d'affect toutefois, sentiment ne peut pas être considéré comme pauvre au plan sémantique. Certains noms comme amour ou amitié se définissent bien, en effet, comme appartenant à la classe des sentiments. Ce fait le distingue d'autres noms généraux comme le 'nom coquille' fait ou les noms mentaux idée, notion qui ne structurent pas des classes ontologiques. De plus, dans la structure un/ce sentiment de, ce dernier a fonction de classifieur. Il fait basculer des noms psychologiques dans le domaine affectif.

Émotion est bien, lui aussi, un nom général, un hyperonyme de la classe des affects, mais au contenu sémantique plus stable : sa combinatoire lexicale est proche de ses hyponymes les plus prototypiques (peur, colère, joie). De ce fait, il ne nécessite pas de complémentation pour spécifier son sens. Il a un emploi anaphorique limité. Son contenu sémantique ne se définit pas par rapport au contexte immédiat, et, de ce fait, il ne peut être considéré comme shell noun, ni comme classifieur.

La comparaison des deux genres textuels nous a permis de mieux faire émerger ce statut spécifique. Ainsi, dans le corpus littéraire, sentiment s'associe davantage avec éprouver, tandis que, dans la presse, c'est l'acception 'impression' qui domine, grâce aux verbes supports avoir et donner. Il attire beaucoup moins de collocatifs pour la manifestation et le contrôle (comparé à émotion), et ce uniquement dans la littérature. Ses emplois de shell noun sont proportionnellement plus courants dans la presse, ce qui pourrait être lié aux liens cohésifs plus caractéristiques pour ce genre.

L'éclairage diachronique permet d'ancrer dans une temporalité plus longue les phénomènes observés autour de sentiment et émotion dans le corpus contemporain. Sur le plan discursif, nous avons pu constater l'ancienneté des emplois de type «emphatique » pour sentiment, ce qui n'est pas le cas d'émotion. Par ailleurs, le fait que sentiment a développé, sur le plan syntaxique, une complémentation plus fréquente, tandis qu'émotion perdait cette possibilité après le $\mathrm{XVII}{ }^{\mathrm{e}} \mathrm{s}$., a contribué au basculement progressif de sentiment vers le statut de classifieur. L'étude des associations lexicales spécifiques des deux noms corrobore cette conclusion en montrant que sentiment a conservé sa combinatoire avec les verbes support, ce qui a sans doute également contribué à son évolution vers le statut de shell noun, contrairement à émotion. Ce dernier a privilégié les collocatifs de la dimension 'manifestation', ce qui s'explique par un changement du contexte culturel : la société qui retenait et cachait ses émotions les affiche de nouveau dans les romans qui mettent en scène des personnages émus. Une des perspectives diachroniques pour prolonger le présent travail pourrait envisager plus finement le rôle joué par les genres littéraires sur l'évolution des noms sentiment et émotion. 


\section{Références}

Anscombre J.-P. (1995), « Morphologie et représentation événementielle : le cas de sentiment et d'attitude », Langue française $105,40-54$.

ARIES P. \& Duby G. (1987), Histoire de la vie privée. De la Révolution à la Grande Guerre. Paris : Seuil.

Augustyn M. \& Grossmann F. (2014), «Entre hyperonymie et spécification : un drôle de sentiment », in P. Blumenthal, I. Novakova \& D. Siepmann (éds), Les émotions dans le discours. Emotions in Discourse, Frankfort sur le Main : P. Lang, 123-134.

Blumenthal P. (2009), «Les noms d'émotions : trois systèmes d'ordre », in I. Novakova \& A. Tutin (éds), Le lexique des émotions, Grenoble : ELLUG, 41-64.

Blumenthal, P. (2012), «Méthodes statistiques en lexicologie contrastive», in L. Begioni \& C. Bracquenier (éds), Sémantique et lexicologie des langues d'Europe, Rennes : Presses Universitaires de Rennes, 114-128.

Buvet P.-A., Girardin C., Gross G. \& Groud C. (2005), «Les prédicats d'<affect> », Lidil 32, 123-143.

Condamines A . (2005), «Anaphore nominale infidèle et hyperonymie : le rôle du genre textuel », Revue de Sémantique et Pragmatique 18, 32-42.

CoRnish, F. (2008), "How Indexicals Function in Texts: Discourse, Text, and one Neo-Gricean Account of Indexical Reference", Journal of Pragmatics 40 (6), 997-1018.

Diwersy S., Goossens V., Grutschus A., Kerne B., Kraif O., Melnikova E. \& Novakova I. (2014), « Traitement des lexies d'émotion dans les corpus et les applications d'EmoBase», Corpus 13, 269-293. Disponible en ligne sur <http://corpus.revues.org/2537> (consulté le 26 mars 2016).

DunNing T. (1993), “Accurate Methods for the Statistics of Surprise and Coincidence”, Computational Linguistics 19.1, 6174.

FERRER V. \& RAMOND C. (éd.) (2017). La langue des émotions. XVIe-XVIII siècle. Paris : Classiques Garnier.

Halliday M. A. K. \& HASSAN R. (1976), Cohesion in English, London: Logman.

Hoey M. (2005), Lexical Priming. A New Theory of Words and Language, London/New York: Routledge.

HuYGHE R. (2015), «Les typologies nominales : présentation », Langue française 185, 5-27.

KRAIF O. \& Diwersy S. (2014), "Exploring Combinatorial Profiles Using Lexicograms on a Parsed Corpus: a Case Study in the Lexical Field of Emotions", in P. Blumenthal, I. Novakova \& D. Siepmann (éds), Les émotions dans le discours. Emotions in Discourse, Frankfort sur le Main : P. Lang, 381-394.

KrzyZANowsKa A. (2014), «Les termes génériques du vocabulaire affectif : le cas de sentiment et uczucie», in P. Blumenthal, I. Novakova \& D. Siepmann (éds), Les émotions dans le discours. Emotions in Discourse, Frankfort sur le Main : P. Lang, 105-120.

Legallois D. (2006), «Quand le texte signale sa structure : la fonction textuelle des noms sous-spécifiés », Corela HS-5. Disponible en ligne sur 〈https://corela.revues.org/1465> (consulté le 02/05/2017).

Novakova I. \& Sorba J. (2014), «L'émotion dans le discours. À la recherche du profil discursif de stupeur et de jalousie », in P. Blumenthal, I. Novakova \& D. Siepmann (éds), Les émotions dans le discours. Emotions in Discourse, Frankfort sur le Main : P. Lang, 161-173.

Novakova I. \& Tutin A. (éds.) (2009), Le Lexique des émotions, Grenoble : ELLUG.

PLANTIN C. (2011). Les bonnes raisons des émotions. Principes et méthode pour l'étude du discours émotionné, Bern : P. Lang.

PR : Le Petit Robert électronique (C) 2016 Dictionnaires Le Robert - Le Petit Robert de la langue française [03/04/2017].

RAMOND C. (2017), «La mimesis dramatique dans quelques romans du XVIII ${ }^{e}$ siècle ou la puissance rêvée de l'émotion directe », in V. Ferrer \& C. Ramond (éds). La langue des émotions. XVI ${ }^{e}$-XVIII ${ }^{e}$ siècle, Paris : Classiques Garnier, 307-325.

Rosenwein B. H., DeBIEs M.-H. \& DejoIS C. (2006), « Histoire de l'émotion : méthodes et approches », Cahiers de civilisation médiévale, $49^{\mathrm{e}}$ année ( $\left.{ }^{\circ} 193\right)$, La médiévistique au XX $X^{e}$ siècle. Bilan et perspectives, 33-48.

Schmid H.-J. (2000), English Abstract Nouns as Conceptual shells: From Corpus to Cognition, Berlin / New York: Mouton de Gruyter.

Sinclair J. (2004), Trust the Text: Language, Corpus and Discourse, London: Routledge.

SOUTET O. (2011), « Avant-propos », Diachroniques. Revue de linguistique française diachronique 1, 7-13.

TLF : Trésor de la langue française. Dictionnaire de la langue du XIXe et du XX siècle (1789-1960). Paris : Éditions du Centre National de la Recherche Scientifique (t. 1-10). Gallimard (t. 11-16). 1971-1994.

Tutin A., Novakova I., Grossmann F. \& Cavalla C. (2006), « Esquisse de typologie des noms d'affect à partir de leurs propriétés combinatoires », Langue française 150, 32-49.

WINTER E. O. (1992). "The notion of unspecific versus specific as one way of analysing the information of a fund-raising letter", in W.C. Mann \& S.A. Thompson (éds), Discourse descriptions: Diverse analyses of a fund-raising text, Amsterdam: John Benjamins, 131-70. 